Article

\title{
Optimizing Sequential Systemic Therapies for Advanced Hepatocellular Carcinoma: A Decision Analysis
}

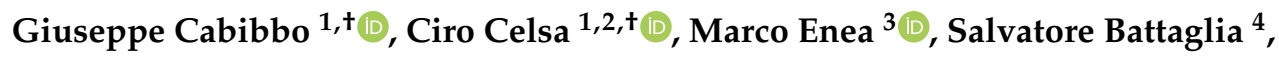 \\ Giacomo Emanuele Maria Rizzo ${ }^{1}$ (D), Stefania Grimaudo ${ }^{1}$ (D), Domenica Matranga ${ }^{3}$, \\ Massimo Attanasio ${ }^{4}$, Paolo Bruzzi ${ }^{5}$, Antonio Craxì ${ }^{1}$ and Calogero Cammà ${ }^{1, *}$ \\ 1 Department of Health Promotion Sciences Maternal and Infant Care, \\ Section of Gastroenterology \& Hepatology, Internal Medicine and Medical Specialties, PROMISE, \\ University of Palermo, 90127 Palermo, Italy; giuseppe.cabibbo78@gmail.com (G.C.); \\ celsaciro@gmail.com (C.C.); g.rizzo.gr@gmail.com (G.E.M.R.); stefania.grimaudo@unipa.it (S.G.); \\ antonio.craxi@unipa.it (A.C.) \\ 2 Department of Surgical, Oncological and Oral Sciences (Di.Chir.On.S.), University of Palermo, \\ 90127 Palermo, Italy \\ 3 Department of Health Promotion Sciences Maternal and Infant Care, \\ Internal Medicine and Medical Specialties, PROMISE, University of Palermo, 90127 Palermo, Italy; \\ marco.enea@unipa.it (M.E.); domenica.matranga@unipa.it (D.M.) \\ 4 Dipartimento di Scienze Economiche, Aziendali e Statistiche, University of Palermo, 90133 Palermo, Italy; \\ salvatore.battaglia91@gmail.com (S.B.); massimo.attanasio@unipa.it (M.A.) \\ 5 U.O. Epidemiologia Clinica, IRCCS Ospedale Policlinico San Martino, 16132 Genova, Italy; \\ bruzzipaolo49@gmail.com \\ * Correspondence: calogero.camma@unipa.it \\ + These authors equally contributed to this work.
}

Received: 30 June 2020; Accepted: 27 July 2020; Published: 31 July 2020

\begin{abstract}
Background: An optimal sequential systemic therapy for advanced hepatocellular carcinoma (HCC) has not been discovered. We developed a decision model based on available clinical trials to identify an optimal risk/benefit strategy for sequences of novel systemic agents. Methods: A Markov model was built to simulate overall survival (OS) among patients with advanced HCC. Three first-line (single-agent Sorafenib or Lenvatinib, and combination of Atezolizumab plus Bevacizumab) followed by five second-line treatments (Regorafenib, Cabozantinib, Ramucirumab, Nivolumab, Pembrolizumab) were compared in fifteen sequential strategies. The likelihood of transition between states (initial treatment, cancer progression, death) was derived from clinical trials. Life-year gained (LYG) was the main outcome. Rates of severe adverse events (SAEs) ( $\geq$ grade 3 ) were calculated. The innovative measure, called incremental safety-effectiveness ratio (ISER), of the two best sequential treatments was calculated as the difference in probability of SAEs divided by LYG. Results: Lenvatinib followed by Nivolumab (median OS, 27 months) was the most effective sequence, producing a LYG of 0.75, while Atezolizumab plus Bevacizumab followed by Nivolumab was the safest sequence (SAEs 40\%). Accordingly, the net health benefit assessed by ISER favored Lenvatinib followed by Nivolumab, compared to Atezolizumab plus Bevacizumab, followed by Nivolumab in $52 \%$ of cases. Conclusion: Further sequential clinical trials or large-scale real-world studies may prove useful to evaluate the net health benefit of the best sequential treatment for advanced HCC.
\end{abstract}

Keywords: hepatocellular carcinoma; systemic therapy; sequential therapy; tumor progression; survival 


\section{Introduction}

Hepatocellular carcinoma (HCC) is characterized by clinical and biological heterogeneity $[1,2]$. In addition, most cases of HCC have a dismal prognosis that is strictly linked to the stage of cirrhosis [3]. Unfortunately, more than half of all cases of HCC are diagnosed at a stage with no potentially curative treatment options. Since 2008, the oral multikinase inhibitor (MKI), Sorafenib, has been recommended as the standard first-line systemic therapy for patients with Barcelona Clinic Liver Cancer (BCLC) C (advanced) HCC, and also for patients with BCLC B (intermediate) HCC, which have been deemed unfit for, or who fail to respond to, loco-regional therapies $[4,5]$. In field practice studies which have assessed the efficacy and cost-effectiveness of Sorafenib for HCC, high rates of treatment discontinuation have occurred, due to tumor progression, liver decompensation, and severe adverse events (SAEs) [6-10].

Until 2018, Sorafenib was the only systemic treatment which was found to significantly improve overall survival (OS) as a first-line therapy for advanced HCC [11]. Subsequently, Lenvatinib was found to be non-inferior to Sorafenib in terms of OS, and it also exhibited significantly better progression-free survival (PFS) in a randomized controlled trial (RCT) [12]. More recently, immune checkpoint inhibitors (ICIs), particularly antibodies targeting the programmed cell death-1 (PD-1)/programmed cell death ligand-1 (PD-L1) pathway, have been a major breakthrough in drug development for HCC [13]. In 2020, combined therapy with Atezolizumab and Bevacizumab has exhibited a significant improvement in OS and PFS compared with Sorafenib [14].

Among patients who have progressed and are tolerant to Sorafenib, treatment with Regorafenib has resulted in longer OS than placebo [15]. Thus, Regorafenib has become the first second-line drug to be approved for HCC [15]. Subsequently, Cabozantinib [16] and Ramucirumab [17] have exhibited a survival benefit versus placebo in second-line phase III RCTs. For Ramucirumab, these results were observed in patients with elevated alpha-fetoprotein. After 2017, the Food and Drug Administration granted accelerated approval to Nivolumab and Pembrolizumab, two monoclonal antibodies which act as inhibitors of PD-1 in a second-line setting [18-22]. More recently, however, Pembrolizumab failed to achieve statistically significant improvement in OS and PFS compared to placebo in second-line setting [23].

To date, the best sequential first- and second-line systemic treatment remains elusive. In particular, what is the best sequential systemic strategy in terms of OS benefit and safety? Due to the lack of sequential appropriately designed RCTs, these issues remain unanswered.

Therefore, we have developed a Markov model based on data from available RCTs, and we describe its use to evaluate the risks and benefits of currently available sequential systemic treatments for patients with advanced HCC.

\section{Results}

The general structure of the Markov model is showed in Figure 1. Outcomes reported in the clinical trials examined and model parameters are reported in Table 1 and Appendix A Tables A1 and A2. The base-case effectiveness and safety of the 15 treatment sequences ordered according to median OS are reported in Table 2. First-line Lenvatinib followed by second-line Nivolumab was the most effective treatment (median OS, 27 months), with a LYG value of 0.75 and a NNT of 5 . In contrast, first-line Sorafenib followed by second-line Ramucirumab was the least effective treatment sequence (median OS, 18 months). Figure 2 shows the simulated survival curves of the three most effective treatment sequences (Lenvatinib followed by Nivolumab, Lenvatinib followed by Pembrolizumab, and Atezolizumab plus Bevacizumab followed by Nivolumab, respectively). At a threshold of 1000 patients per sequence, the simulated estimates of median OS were significantly higher for Lenvatinib followed by Nivolumab, compared to Lenvatinib followed by Pembrolizumab $(p=0.002)$ and Atezolizumab plus Bevacizumab followed by Nivolumab $(p=0.035)$ (Supplementary Table S1). Survival curves for the other sequences are shown in Supplementary Figures S1-S3.

Among the 15 treatment sequences analyzed, the treatment sequence with the lowest rate of SAEs was first-line Atezolizumab plus Bevacizumab followed by second-line Nivolumab (40.2\%). 
Conversely, the treatment sequences exhibiting the highest toxicity (77.2\% SAEs) were first-line Lenvatinib followed by second-line Cabozantinib or Regorafenib. (Table 2).

Next, the most effective sequence (Lenvatinib followed by Nivolumab) was compared with the safest sequence (Atezolizumab plus Bevacizumab followed by Nivolumab). A one-way sensitivity analysis for LYG was performed for these two sequences in comparison with Sorafenib followed by Nivolumab. LYG ranged from 0.25 to 0.50 for Lenvatinib followed by Nivolumab, and from 0 to 0.25 for Atezolizumab plus Bevacizumab followed by Nivolumab. The most influential factors identified for both strategies were: HBV infection, HCV infection, MaVI and/or EHD, ECOG-PS 1, and BCLC stage $C$ (Figure 3). Variations of $\pm 30 \%$ in the distribution of these variables had a greater influence on their effectiveness.

Table 1. Outcomes reported in the clinical trials examined and model parameters.

\begin{tabular}{|c|c|c|}
\hline Variables & Base-Case & References \\
\hline First-line systemic treatments & & \\
\hline \multicolumn{3}{|l|}{ Median PFS (months) reported in RCTs } \\
\hline Sorafenib & $3.8 \mathrm{mo}$ & {$[12,14,24-27]$} \\
\hline Lenvatinib & $7.4 \mathrm{mo}$ & [12] \\
\hline Atezolizumab plus Bevacizumab & $6.8 \mathrm{mo}$ & [14] \\
\hline \multicolumn{3}{|c|}{ Median PFS (IQR) (months) obtained with Weibull distribution } \\
\hline Sorafenib & $4.6(6.7) \mathrm{mo}$ & \\
\hline Lenvatinib & $8.8(11.7) \mathrm{mo}$ & \\
\hline Atezolizumab plus Bevacizumab & $7.2(9.3) \mathrm{mo}$ & \\
\hline \multicolumn{3}{|l|}{ SAEs } \\
\hline Sorafenib & $72.3 \%$ & {$[12,14,24,25]$} \\
\hline Lenvatinib & $75 \%$ & [12] \\
\hline Atezolizumab plus Bevacizumab & $61 \%$ & [14] \\
\hline \multicolumn{3}{|l|}{ Second-line systemic treatments } \\
\hline \multicolumn{3}{|l|}{ Median OS (months) reported in RCTs } \\
\hline Regorafenib & $10.6 \mathrm{mo}$ & [15] \\
\hline Cabozantinib & $10.2 \mathrm{mo}$ & [16] \\
\hline Ramucirumab & $9.2 \mathrm{mo}$ & [28] \\
\hline Nivolumab & $15.1 \mathrm{mo}$ & [20] \\
\hline Pembrolizumab & $13.9 \mathrm{mo}$ & [23] \\
\hline \multicolumn{3}{|c|}{ Median OS (IQR) (months) obtained with Weibull distribution } \\
\hline Regorafenib & $11.4(14.4) \mathrm{mo}$ & \\
\hline Cabozantinib & $11.4(14) \mathrm{mo}$ & \\
\hline Ramucirumab & $11.3(14.5) \mathrm{mo}$ & \\
\hline Nivolumab & $16.4(23.2) \mathrm{mo}$ & \\
\hline Pembrolizumab & $14(17.8) \mathrm{mo}$ & \\
\hline \multicolumn{3}{|l|}{ SAEs } \\
\hline Regorafenib & $79.7 \%$ & [15] \\
\hline Cabozantinib & $79.4 \%$ & [16] \\
\hline Ramucirumab & $70.4 \%$ & [28] \\
\hline Nivolumab & $18.6 \%$ * & [20] \\
\hline Pembrolizumab & $52.7 \%$ & [23] \\
\hline
\end{tabular}

OS, overall survival. PFS, Progression-free survival. mo, months. IQR, Interquartile Range. SD, Standard Deviation. SAEs, severe adverse events. BCLC, Barcelona Clinic Liver Cancer. RCTs, randomized controlled trials. For Sorafenib, data were pooled from the available RCTs $[14,16,24-27] .{ }^{*}$ Because the incidence of treatment emergent AEs was not reported for Nivolumab, we reported severe treatment-related adverse events. 
Table 2. Base-case effectiveness and safety of 15 treatment sequences according to median OS.

\begin{tabular}{|c|c|c|c|c|c|}
\hline Treatment Sequence & Median OS (mo) & NNT & 36-Month OS (\%) & LYG (yr) & SAEs (\%) \\
\hline Lenvatinib-Nivolumab & 27 & 5 & 32.9 & 0.75 & 48.0 \\
\hline Lenvatinib-Pembrolizumab & 25 & 7 & 26.8 & 0.58 & 64.4 \\
\hline Atezolizumab plus Bevacizumab-Nivolumab & 24 & 6 & 29.5 & 0.50 & 40.2 \\
\hline Sorafenib-Nivolumab & 23 & 7 & 26.1 & 0.42 & 46.3 \\
\hline Atezolizumab plus Bevacizumab-Pembrolizumab & 23 & 9 & 23.4 & 0.42 & 57.1 \\
\hline Lenvatinib-Ramucirumab & 22 & 13 & 19.9 & 0.33 & 72.8 \\
\hline Lenvatinib-Regorafenib & 22 & 14 & 19.3 & 0.33 & 77.2 \\
\hline Lenvatinib-Cabozantinib & 22 & 14 & 19.2 & 0.33 & 77.2 \\
\hline Sorafenib-Pembrolizumab & 20 & 15 & 18.9 & 0.17 & 62.9 \\
\hline Atezolizumab plus Bevacizumab-Ramucirumab & 20 & 23 & 16.7 & 0.17 & 65.6 \\
\hline Atezolizumab plus Bevacizumab-Regorafenib & 20 & 29 & 15.8 & 0.17 & 70.0 \\
\hline Atezolizumab plus Bevacizumab-Cabozantinib & 20 & 30 & 15.6 & 0.17 & 69.9 \\
\hline Sorafenib-Cabozantinib & 18 & 83 & 11.1 & 0.00 & 75.8 \\
\hline Sorafenib-Regorafenib & 18 & 200 & 11.8 & 0.00 & 75.9 \\
\hline Sorafenib-Ramucirumab & 18 & - & 12.3 & 0.00 & 71.4 \\
\hline
\end{tabular}

LYG, life-year gained. All the sequences were compared to the worst sequence (Sorafenib-Ramucirumab). OS, overall survival. SAEs, severe adverse events. NNT, number needed to treat. yr, years. mo, months.

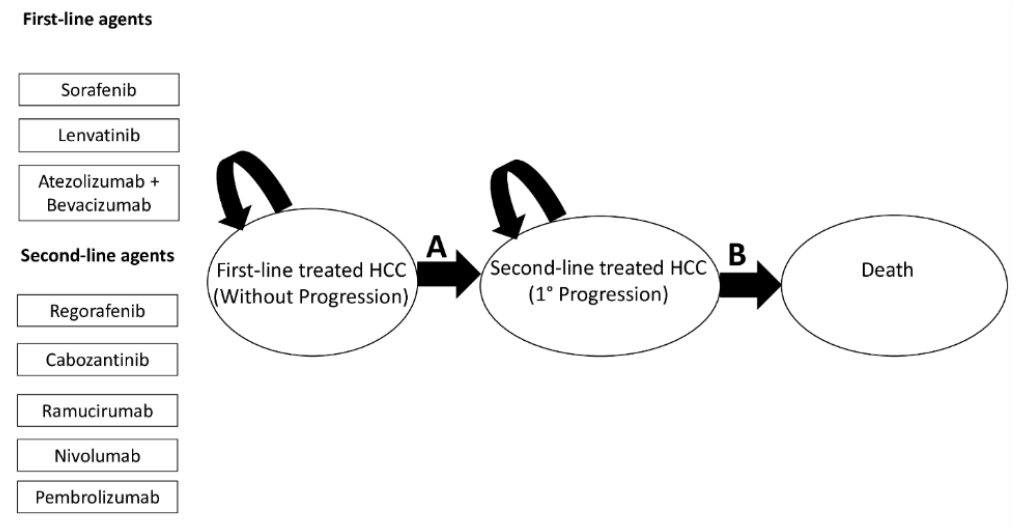

A: first-line progression-free survival. B: second-line overall survival.

Figure 1. General structure of the Markov model used.

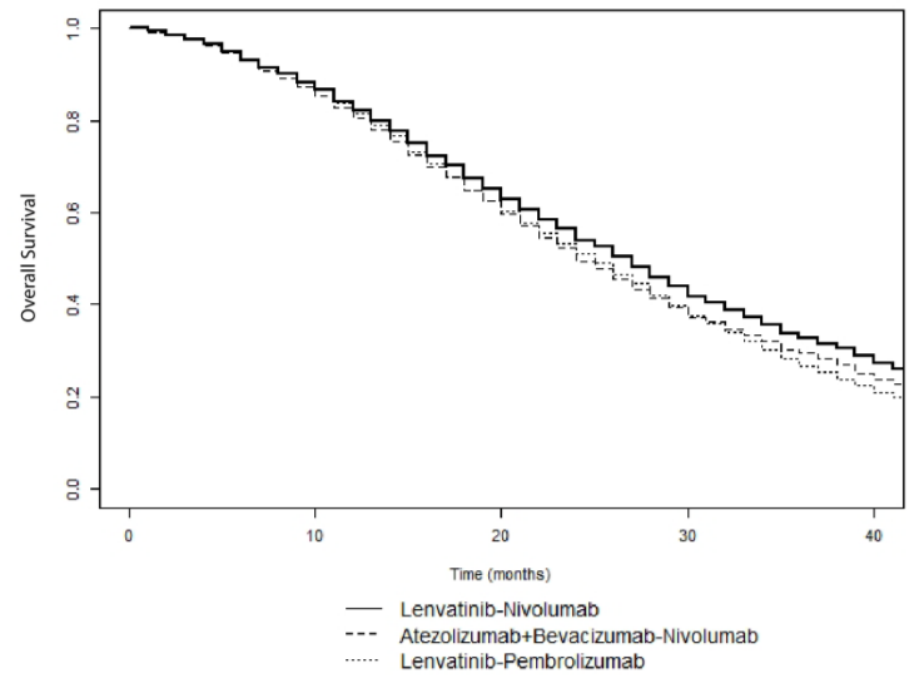

Figure 2. Simulated survival curves of Lenvatinib followed by Nivolumab, Lenvatinib followed by Pembrolizumab, and Atezolizumab plus Bevacizumab followed by Nivolumab sequences in patients with advanced HCC. 
A

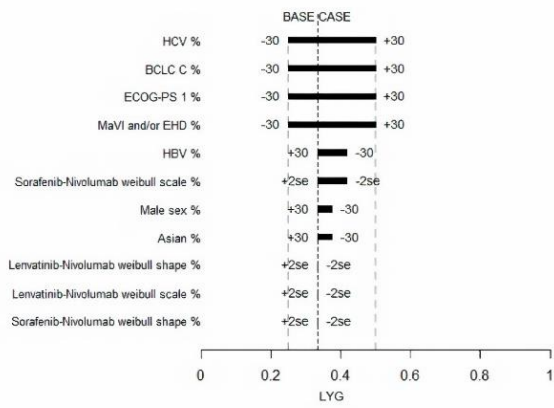

B

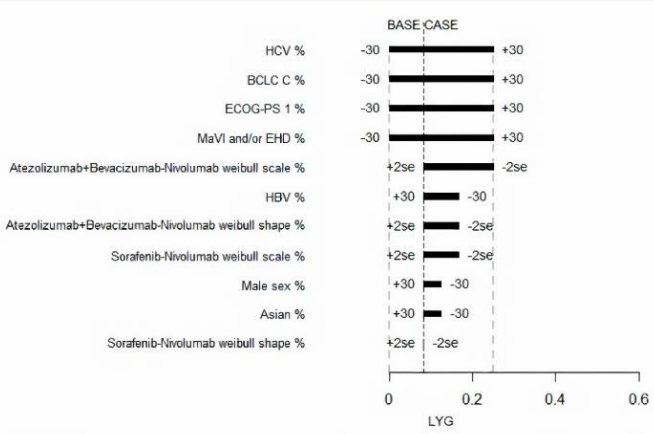

Figure 3. Tornado diagram for the one-way sensitivity analysis of variables influencing LYG of advanced HCC patients treated with Lenvatinib-Nivolumab (A) or Atezolizumab plus Bevacizumab-Nivolumab (B). Both were compared to the Sorafenib-Nivolumab treatment.

Compared to Atezolizumab plus Bevacizumab followed by Nivolumab, the ISER of Lenvatinib followed by Nivolumab was 32\% of SAEs for LYG. One-way sensitivity analysis of the ISER also showed that MaVI and/or EHD was the most influential factor, producing an ISER ranging from 32-94\% of SAEs for LYG. (Supplementary Figure S4).

A two-way sensitivity analysis was performed to demonstrate which therapy would be preferred for varying the difference in SAE and LYG values (Figure 4 and Supplementary Figure S5). At a willingness-to-risk threshold of 30\% of SAEs for LYG, first-line Lenvatinib followed by second-line Nivolumab was favored in 52\% of cases overall. For instance, in scenario B (high LYG and high SAEs), Lenvatinib followed by Nivolumab was favored in 74\% of cases, while in scenario C (low LYG and low SAEs), it was favored in only $36 \%$ of cases.

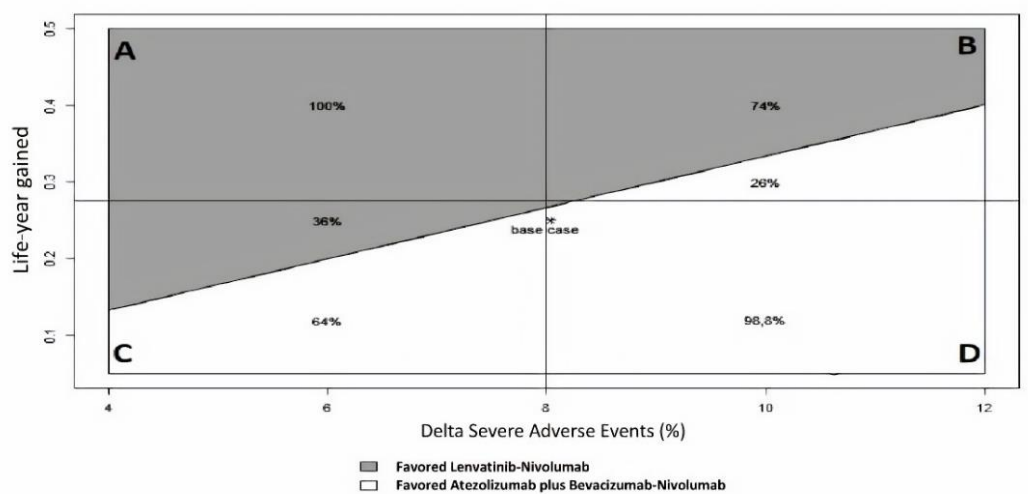

Figure 4. Two-way sensitivity analysis of ISER (Delta Severe Adverse Events\%/Life-year gained) to indicate which therapy is favored for varying Severe Adverse Events (SAEs) and LYG (Life-Year Gained) values, according to a "willingness-to-risk" threshold of 30\% of SAEs for LYG. Grey area favored Lenvatinib-Nivolumab, while white area favored Atezolizumab plus Bevacizumab-Nivolumab. Four clinical scenarios were modeled: (A) high LYG, low SAEs; (B) high LYG, high SAEs; (C) low LYG, low SAEs; (D) low LYG, high SAEs. 
The results of a probabilistic analysis for LYG are presented in Figure S6. In the Monte Carlo simulation, the varying prevalence of HBV infection, HCV infection, MaVI and/or EHD, and ECOG-PS 1 simultaneously, Lenvatinib followed by Nivolumab, and Atezolizumab plus Bevacizumab followed by Nivolumab, were more effective than Sorafenib followed by Nivolumab in $100 \%$ and $92 \%$ of the simulations, respectively.

\section{Discussion}

It is well known that sequential trials in oncology are difficult to perform, and in most settings, such as advanced HCC, they are practically unfeasible. Moreover, real-world data of sequential therapies in advanced HCC are time consuming and they are lacking to date. Therefore, comparative evidence among different treatment sequences could be obtained only by simulation models. In the field of HCC, more innovative treatments are found to confer significant survival benefits, even when used in the advanced stages of disease. To our knowledge, this is the first model to date which provides a comprehensive comparison of different sequential strategies for patients with advanced or intermediate HCC, who are unfit for, or who do not respond to, loco-regional therapies.

Our model suggests that first-line Lenvatinib followed by second-line Nivolumab or Pembrolizumab may be an attractive sequential systemic strategy in terms of OS for patients with advanced HCC. Meanwhile, first-line Atezolizumab plus Bevacizumab followed by second-line Nivolumab was identified as the safest sequential strategy. Strategies involving first-line MKI followed by second-line MKI appear to be associated with worse survival in all scenarios. Using a willingness-to-risk threshold of $30 \%$ of SAEs for LYG, the net health benefit assessed by ISER favored Lenvatinib followed by Nivolumab compared to Atezolizumab plus Bevacizumab, followed by Nivolumab in $52 \%$ of cases, giving a wide range of uncertainty about the net health benefit between the two compared sequences.

RCTs of systemic therapies for advanced HCC have often showed small, albeit significant, differences in OS between active drug and placebo. If the OS gain is relatively small (on the order of a few months), the quality of life spent on or off active treatment is crucial, and it should be considered in the clinical decision-making process. Hence, our model provides evidence that firstand second-line ICIs are the safest sequential strategies. Moreover, sequential first-line ICIs should be reserved for the most fragile patients, considering the relatively improved safety profile they exhibit compared to MKIs. However, ICIs are associated with immune-mediated hepatotoxicity whose severity is unpredictable. The hepatotoxicity can range from mild to life-threatening, and it is not completely known in patients with cirrhosis $[29,30]$.

According to ASCO statements [31], net health benefit is defined as the balance between clinical benefit and SAEs, and it is used to assess the magnitude of the difference between two different therapeutic strategies [32]. In this model, we proposed an innovative measure that combines effectiveness and safety, called ISER. Given the wide range of uncertainty on the net health benefit assessed by ISER between Lenvatinib followed by Nivolumab compared to Atezolizumab plus Bevacizumab followed by Nivolumab, the best treatment strategy should be carefully agreed upon, with the policy makers also taking into account different factors, including the cost-effectiveness.

The results obtained in the present study appear to be plausible and are supported by other evidence. A study of tumor microenvironment and immune setting in patients treated with Sorafenib has revealed a possible role for MKIs also in immunomodulation [33]. Furthermore, Sorafenib treatment may increase the expression of PD-L1 in tumor-infiltrating immune cells [33]. While the expression of PD-L1 in tumor microenvironments has been evaluated as a predictive biomarker for immunotherapy in other solid tumors (i.e., melanoma, non-small cell lung cancer), the CheckMate 040 trial assessing Nivolumab as a second-line treatment for HCC did not find any differences in cancer response according to PD-L1 expression in tumor cells [19]. However, PD-L1 expression was not assessed in tumor-infiltrating immune cells which are involved in immune-surveillance, and probably in HCC progression, after Sorafenib treatment. Additional evidence involves a potential synergistic role for Lenvatinib with ICIs, thereby inhibiting an immunosuppressive microenvironment and promoting 
anti-tumor immune activation [34]. Mounting evidence from preclinical and clinical studies supports the use of immunotherapy as a second-line treatment after locoregional treatments, such as thermoablation, to increase the antitumor immune response [35]. It has been proposed that hyperthermia may activate quiescent dendritic cells and improve leukocyte trafficking, thereby enhancing the effectiveness of subsequent immunotherapy. Finally, it should be considered that only few data exist in the Imbrave 150 [14] and in KeyNote 240 [23] RCTs regarding the sequential treatment of first-line ICIs followed by second-line ICIs, and that it may not have a strong biological plausibility. However, the sequence ICIs-ICIs was used in other oncological settings, i.e., Ipilimumab followed by Nivolumab was used in patients with advanced melanoma [36,37].

There are several limitations associated with our model.

(1) The biggest and most important issue of our decision model is that it did not consider the carry-over effect. Further sequential trials with a crossover design, properly designed to assess the carryover effect, could solve this intriguing issue.

(2) The lack of clinical data concerning the majority of sequential strategies assessed by our model. All the five available second-line therapies were approved in trials, including Sorafenib-experienced patients, while no second-line therapy was evaluated after Lenvatinib or Atezolizumab plus Bevacizumab. As Atezolizumab plus Bevacizumab will become the new standard first-line treatment of advanced HCC, the identification of the optimal treatment sequential strategy represents a burning unsolved medical need. Therefore, our decision model, with all the limitations of a simulation, should be considered a useful tool to evaluate the risk-benefit ratio of treatment sequences not evaluable in the trials performed to date. In the absence of sequential RCTs, large-scale real-world studies are urgently needed.

(3) Trials of second-line treatment included in the model had some differences in inclusion and exclusion criteria, particularly regarding MKIs. However, we are confident that the patients enrolled in second-line RCTs that we examined could be roughly comparable, because all of the patients were Sorafenib-experienced.

(4) Assessment of the benefit of ICIs raises several issues considering the unconventional pattern of radiological response or progression. Novel radiological response assessment tools (i.e., immuneRECIST) and innovative methodologies (i.e., Weibull distribution, restricted mean survival time, landmark analyses) may be able to capture the long-term benefit observed with ICIs. In addition, the use of multivariate meta-analysis, which combines surrogate endpoints (i.e., PFS and objective response rate) with OS, may prove useful for substantiating the benefit of different sequential treatments on OS.

(5) Lack of data regarding all-cause SAEs for Nivolumab may have affected our analyses. However, reported data for Pembrolizumab led to a more accurate assessment of overall tolerability of ICIs in patients with liver disease [30].

(6) Unfortunately, we were unable to calculate quality-adjusted life-years (QALYs), since utilities were not available in the majority of the RCTs examined, particularly regarding ICI trials. Accordingly, we assessed LYG as the primary measure of efficacy. However, utilities may vary widely across different patient subgroups, and they critically depend on quality of life assumptions.

(7) Lack of data on potentially relevant prognostic factors (i.e., microscopic vascular invasion, histological grading, gene profiling [2]), and liver decompensation, that is, a well-known driver of death in HCC patients [38,39], may have affected our results.

The main strengths of our study are that: (1) this is the first model to estimate survival in sequential therapies by using PFS for first-line therapy and OS for second-line therapy. Simulation remains today the only method to provide quantitative estimates for physicians and policymakers in the setting of advanced HCC; (2) it provides a novel method to estimate the net health benefit by the use of the innovative measure, so-called ISER. 


\section{Materials and Methods}

\subsection{Decision Model}

A semi-Markov model was developed to analyze the effectiveness of various sequential systemic treatments for advanced HCC over a lifetime horizon (Figure 1), and to allow the transition hazard to increase over time. This approach is parametric, and it was performed by estimating the scale and shape parameter of a Weibull distribution. Natural mortality rate at age of population was included in the model for a sixty-year-old hypothetical male patient. Given the very short life expectancy, we did not discount risks and benefits. The sequential semi-Markov model was built by using an ad hoc routine written with $\mathrm{R}$ statistical software. We simulated the clinical history of a hypothetical cohort of patients with advanced HCC, which had the same characteristics as patients enrolled in clinical trials [12,14-16,20,23-28] (see Tables 1 and A1). PFS was used as the main endpoint for first-line treatments, because it is not influenced by post-progression survival, and because it is a validated surrogate for OS in advanced HCC [40]. In addition, data describing OS and PFS treatment benefits were derived from Kaplan-Meier curves from the above-mentioned trials. In particular, PFS curves of patients receiving Sorafenib as a first-line treatment were extracted from reports of six phase III RCTs [12,14,24-27]. For Lenvatinib and Atezolizumab plus Bevacizumab, PFS curves were extracted from the REFLECT trial [12] and the IMbrave150 trial [14], respectively. OS curves for patients treated with second-line therapies were extracted from RESORCE [15], CELESTIAL [16], REACH [28], CheckMate-040 [20], and KeyNote-240 [23] trials. Individual patient survival data were reconstructed by using an algorithm proposed by Guyot et al. [41] This algorithm provides a list of patients with predicted survival times and a predicted event of interest (i.e., alive or dead; progression or no progression) by using digitalized data on survival probabilities, time, and total number of patients and events. Engauge Digitizer software Version 12 was used to extract data from the curves. Each reconstructed survival curve was inspected for accuracy and was compared with originally published curves. Supplementary Figures S7-S14 show the Kaplan-Meier curves which were reconstructed from published curves, with estimated Weibull survival curves superimposed. The scale and shape parameter of a Weibull distribution on the data extracted from Kaplan-Meier curves are showed in Table A2. The impact of alternative survival distributions (lognormal, log-logistic, exponential, gamma, generalized gamma) on predicted survival probability was also explored, although none of the considered distributions resulted in a suitable alternative to the Weibull distribution. The lognormal, log-logistic, and exponential distributions were excluded a priori, because they do not allow monotonically increasing hazards to fit, which is a fundamental assumption for the type of disease being evaluated. Similarly, although the gamma and generalized gamma distributions could theoretically allow monotonically increasing hazards, these were first fitted and then excluded after a visual inspection, which showed decreasing (gamma) or dome-shaped (generalized gamma) hazards. $p$-values $\leq 0.05$ were considered statistically significant. As a safety measure, the number of SAEs (grade $\geq 3$ ) from each included trial was extracted. The model evaluated 15 sequential therapy scenarios (Figure S15).

\subsection{Model Transitions and Survival Estimates}

The model simulated transitions among the following three health states, with a cycle length of one month: (1) advanced HCC eligible to first-line systemic therapy; (2) first progression; and (3) death (Figure 1). The health states were mutually exclusive (i.e., a patient could experience a single health state at any given time). Survival estimates for sequential settings considered the proportion of patients who did not receive second-line therapy due to death during first-line therapy. Furthermore, transitions of patients between health states were based on calculated transition probabilities from PFS and OS data extracted from published studies. Simulation of 2000 pseudo-random patients was performed to obtain overall simulated survival times and median times throughout the three states of the disease. 


\subsection{Outcomes}

Life-year gained (LYG) was the main health outcome. Median OS obtained from the Markov model at different simulated sample sizes was compared by using the Wilcoxon test, in order to identify the minimum sample size needed to assess a statistically significant difference. To provide long-term survival information, a milestone survival analysis at 36 months was performed. Accordingly, the number needed to treat (NNT) was calculated for each sequential strategy.

The rate of SAEs ( $\geq$ grade 3 ) was calculated for each sequential treatment, by taking into account transition probabilities from first- to second-line treatments, by using a weighted mean of the number of simulated patients transited in each disease state (initial treatment and cancer progression). To obtain a net health benefit measure [31], clinical benefit and toxicity were combined to calculate a novel measure, incremental safety-effectiveness ratio (ISER). The latter was defined as the difference in the rate of SAEs between two sequential treatments, divided by their difference in effectiveness, and measured in LYG. This unit of measure expresses the incremental percentage of SAEs for each LYG.

$$
I S E R=\frac{\text { Delta } S A E s \%}{L Y G}
$$

\subsection{Deterministic Sensitivity Analysis}

Two different deterministic sensitivity analyses were performed for LYG and ISER. The following variables were evaluated: male gender, Asian ethnicity, hepatitis $C$ virus (HCV) and hepatitis B virus (HBV) as etiology of liver disease, macrovascular invasion (MaVI) and/or extrahepatic disease (EHD), Eastern Cooperative Oncology Group (ECOG) Performance Status 1, and Barcelona Clinic Liver Cancer (BCLC) stage C. (see Appendix A Table A2) We considered \pm two times the standard error (SE) to be the variation range for both the Weibull scale and shape parameters. We considered $\pm 30 \%$ variation for the remaining variables. The latter was actually corrected for the maximum possible variation (i.e., a $30 \%$ increase in MAVI was impossible, as the baseline value was $88 \%$, so a $12 \%$ increase was considered, up to $100 \%$ of MAVI).

In the one-way deterministic sensitivity analysis for LYG, Lenvatinib followed by Nivolumab and Atezolizumab plus Bevacizumab followed by Nivolumab were indirectly compared to Sorafenib followed by Nivolumab.

In the one-way deterministic sensitivity analysis for ISER, Lenvatinib followed by Nivolumab versus Atezolizumab plus Bevacizumab followed by Nivolumab was compared, because the latter dominates Sorafenib followed by Nivolumab, both in terms of median survival and SAEs.

Two-way sensitivity analysis for ISER was performed by varying the numerator (percentage difference in SAEs) and the denominator (LYG). The variation range for the denominator was chosen to be as large as the maximal value observed, while a $\pm 4 \%$ variation was chosen for the numerator. Analogous to the willingness-to-pay aspect of a cost-effectiveness analysis, a willingness-to-risk threshold value must also be established for ISER. Several possible willingness-to-risk thresholds for ISER, and their visual effect in terms of estimated areas, when SAEs and LYG are varied according to their ranges, have been calculated.

\subsection{Probabilistic Sensitivity Analysis}

To examine the extent to which differences in LYG could be explained by differences in patient characteristics, a two-step probabilistic sensitivity analysis was performed. In the first step, four variables (i.e., proportion of patients with HCV infection, HBV infection, ECOG-Performance Status 1, and MacroVascular Invasion and/or extrahepatic disease) were included in a meta-regression model. The dependent variable was median PFS time. The meta-regression model employed was a weighted multiple linear regression model, where the weight was the study sample sizes. By assuming a uniform distribution for the values of the four variables considered, 1000 pseudo-random values were generated from each variable, in order to obtain corresponding predictions from the model. 
In the second step, the predicted median times were then proportionally translated into Weibull scale parameters by assuming, in percentage, a proportional shift of the scale parameters for the base case (hazard proportionality is a property of the Weibull distribution). Then, in turns, these parameters were used as inputs in the Markov model, to obtain 1000 simulated median times.

\section{Conclusions}

Single-agent first-line Lenvatinib, followed by second-line Nivolumab or Pembrolizumab, seemed to be an attractive option for advanced HCC. Meanwhile, the first-line combination of Atezolizumab plus Bevacizumab, followed by second-line Nivolumab, was the safest sequential strategy. Congruent with the above data, a wide range of uncertainty remains about what is the optimal sequential strategy in terms of net health benefit. Ongoing and future RCTs and data obtained in field practice studies will confirm whether combination or sequential systemic therapies are more beneficial. In the meantime, clinicians, patients and policymakers can use this decision model for the collaborative decision-making process.

Supplementary Materials: The following are available online at http://www.mdpi.com/2072-6694/12/8/2132/s1, Figure S1. Simulated survival curves of sequential systemic treatment strategies in patients with advanced hepatocellular carcinoma (first-line Sorafenib); Figure S2. Simulated survival curves of sequential systemic treatment strategies in patients with advanced hepatocellular carcinoma (first-line Lenvatinib); Figure S3. Simulated survival curves of sequential systemic treatment strategies in patients with advanced hepatocellular carcinoma (first-line Atezolizumab plus Bevacizumab); Figure S4. Tornado diagram for a one-way sensitivity analysis of variables which influenced the incremental safety-effectiveness ratio (ISER) of Lenvatinib followed by Nivolumab compared to Atezolizumab + Bevacizuab followed by Nivolumab in patients with advanced hepatocellular carcinoma; Figure S5. Contour plot of ISER for varying delta SAE and LYG values; Figure S6. Empirical cumulative density function curves representing the probability that Lenvatinib followed by Nivolumab or Atezolizumab plus Bevacizuab followed by Nivolumab are more effective than Sorafenib followed by Nivolumab in patients with advanced hepatocellular carcinoma Figure S7: Kaplan-Meier curve with Weibull estimated curve for Sorafenib treatment arms superimposed; Figure S8: Kaplan-Meier curve with Weibull estimated curve for Lenvatinib superimposed; Figure S9. Kaplan-Meier curve with Weibull estimated curve for Atezolizumab + Bevacizumab superimposed; Figure S10. Kaplan-Meier curve with Weibull estimated curve for Regorafenib superimposed; Figure S11. Kaplan-Meier curve with Weibull estimated curve for Cabozantinib superimposed; Figure S12. Kaplan-Meier curve with Weibull estimated curve for Ramucirumab superimposed; Figure S13. Kaplan-Meier curve with Weibull estimated curve for Nivolumab superimposed; Figure S14. Kaplan-Meier curve with Weibull estimated curve for Pembrolizumab superimposed; Figure S15. Fifteen sequential systemic treatment strategies deriving from three first-line and five second-line treatments for patients with advanced HCC; TableS1. Comparison of median overall survival (OS) between Lenvatinib followed by Nivolumab and Atezolizumab + Bevacizumab followed by Nivolumab. The simulated sample size $(\mathrm{N})$ was varied for each treatment arm

Author Contributions: G.C., C.C. (Ciro Celsa), M.E., S.B., S.G., G.E.M.R., D.M., M.A., P.B., A.C. and C.C. (Calogero Cammà) take full responsibility for the study design, data analysis and interpretation, and preparation of the manuscript. All authors were involved in planning the analysis and drafting the manuscript. All authors have read and agreed to the published version of the manuscript.

Funding: This research received no external funding.

Conflicts of Interest: G.C.: participated in advisory board for Bayer and Ipsen. A.C.: Research support from Abbvie, BMS, Gilead, Merck/MSD, Intercept, provided consultancy, speakers bureau and participated in advisory boards for Abbvie, BMS, Gilead, MSD/Merck. C.C. participated in advisory board for Bayer, MSD/Merck and Eisai. The other authors declare no conflict of interest. 


\section{Appendix A}

Table A1. Characteristics of the clinical trials from which model data were extracted.

\begin{tabular}{|c|c|c|c|c|c|c|c|c|}
\hline Trial & Treatments & $\begin{array}{l}\mathrm{N} \text { of Patients in } \\
\text { Each Arm }\end{array}$ & Characteristics of Included Patients & $\begin{array}{c}\text { Median OS } \\
\text { (mo.) }\end{array}$ & $\begin{array}{l}\text { Median PFS } \\
\text { (mo.) }\end{array}$ & $\begin{array}{l}\text { Median TTP } \\
\text { (mo.) }\end{array}$ & ORR $(\%)$ & SAEs (\%) \\
\hline \multicolumn{9}{|c|}{ First-line } \\
\hline REFLECT [12] & Sorafenib; Lenvatinib & $476 ; 478$ & $\begin{array}{l}\text { Unresectable HCC BCLC B or C; } \\
\text { Child-Pugh A; ECOG-PS } 0 \text { or } 1 \text {; one or more } \\
\text { measurable target lesions. Patients with } 50 \% \\
\text { or higher liver occupation, invasion of the } \\
\text { bile duct, or invasion at the main portal vein } \\
\text { and patients previously treated with } \\
\text { systemic therapy were excluded. }\end{array}$ & $12.3 ; 13.6$ & $3.7 ; 7.4$ & $3.7 ; 8.9$ & $12.4 ; 40.6$ & $66.5 ; 75$ \\
\hline IMBrave150 [14] & $\begin{array}{c}\text { Sorafenib; } \\
\text { Atezolizumab+Bevacizumab }\end{array}$ & $165 ; 336$ & $\begin{array}{c}\text { Locally advanced or metastatic } \\
\text { and/or unresectable HCC; No prior } \\
\text { systemic therapy. }\end{array}$ & 13.2; NE & $4.3 ; 6.8$ & - & $12 ; 27$ & $60.9 ; 61$ \\
\hline SUN1170 [35] & Sorafenib; Sunitinib & $544 ; 530$ & $\begin{array}{c}\text { Histologically confirmed, locally advanced } \\
\text { or metastatic HCC candidate for systemic } \\
\text { anticancer treatment. Child-Pugh A; } \\
\text { ECOG-PS } 0 \text { or } 1\end{array}$ & $10.2 ; 7.9$ & $3.0 ; 3.6$ & $3.8 ; 4.1$ & $6.1 ; 6.6$ & $74.2 ; 82.1$ \\
\hline SIRveNIB [36] & Sorafenib;Y90 & $178 ; 182$ & $\begin{array}{l}\text { Locally advanced cancer (BCLC) stage B or } \\
\text { C without extrahepatic disease with or } \\
\text { without portal vein thrombosis, not } \\
\text { amenable to curative treatment modalities. } \\
\text { No more than } 2 \text { previous hepatic } \\
\text { artery-directed treatments. }\end{array}$ & $10.0 ; 8.8$ & $5.1 ; 5.8$ & $5.4 ; 6.1$ & - & $50.6 ; 27.7$ \\
\hline SILIUS [37] & Sorafenib; HAIC & $103 ; 103$ & $\begin{array}{l}\text { Advanced HCC (four or more tumours } \\
\text { refractory to transarterial } \\
\text { chemoembolisation or tumours with } \\
\text { vascular invasion or extrahepatic spread) } \\
\text { not candidates for hepatectomy, local } \\
\text { ablation or transarterial chemoembolisation; } \\
\text { Child-Pugh A-B7; ECOG-PS 0-1 }\end{array}$ & $11.5 ; 11.8$ & $3.5 ; 4.8$ & $3.5 ; 5.3$ & $18 ; 36$ & - \\
\hline SARAH [38] & Sorafenib; $Y 90$ & $222 ; 459$ & $\begin{array}{l}\text { Locally advanced HCC BCLC C or new } \\
\text { HCC not eligible for surgical resection, liver } \\
\text { transplantation, or thermal ablation after a } \\
\text { previously cured HCC (cured by surgery or } \\
\text { thermoablative therapy), or HCC with two } \\
\text { unsuccessful rounds of transarterial } \\
\text { chemoembolization: Child-Pugh A-B7; } \\
\text { ECOG-PS 0-1 }\end{array}$ & $9.9 ; 8.0$ & $3.7 ; 4.1$ & - & $12 ; 19$ & - \\
\hline
\end{tabular}


Table A1. Cont.

\begin{tabular}{|c|c|c|c|c|c|c|c|c|}
\hline Trial & Treatments & $\begin{array}{l}\mathrm{N} \text { of Patients in } \\
\text { Each Arm }\end{array}$ & Characteristics of Included Patients & $\begin{array}{c}\text { Median OS } \\
\text { (mo.) }\end{array}$ & $\begin{array}{c}\text { Median PFS } \\
\text { (mo.) }\end{array}$ & $\begin{array}{c}\text { Median TTP } \\
\text { (mo.) }\end{array}$ & ORR (\%) & SAEs $(\%)$ \\
\hline \multicolumn{9}{|c|}{ Second-line } \\
\hline RESORCE [15] & Regorafenib; Placebo & $379 ; 194$ & $\begin{array}{l}\text { HCC BCLC B or C not amenable to curative } \\
\text { treatment; Child-Pugh A; ECOG-PS } 0 \text { or } 1 ; \\
\text { documented radiological progression } \\
\text { during Sorafenib treatment; tolerance to } \\
\text { Sorafenib ( } \geq 400 \mathrm{mg} \text { daily for at least } 20 \text { of } \\
\text { the } 28 \text { days before discontinuation); last } \\
\text { Sorafenib dose within } 10 \text { weeks of } \\
\text { randomization. Patients with previous } \\
\text { systemic treatment for HCC or } \\
\text { discontinuation of Sorafenib for toxicity } \\
\text { were excluded. }\end{array}$ & $10.6 ; 7.8$ & $3.1 ; 1.5$ & $3.2 ; 1.5$ & $11 ; 4$ & $79.7 ; 58.5$ \\
\hline CELESTIAL [16] & Cabozantinib; Placebo & $470 ; 237$ & $\begin{array}{l}\text { HCC not amenable to curative Treatment; } \\
\text { Child-Pugh class A; ECOG-PS } 0 \text { or } 1 \\
\text { previous treatment with Sorafenib; disease } \\
\text { progression after at least one systemic } \\
\text { treatments (up to 2) }\end{array}$ & $10.2 ; 8.0$ & $5.2 ; 1.9$ & - & $4 ; 1$ & $79.4 ; 48.1$ \\
\hline REACH [39] & Ramucirumab; Placebo & $283 ; 282$ & $\begin{array}{l}\text { BCLC C or B refractory or not amenable to } \\
\text { locoregional therapy; Child-Pugh A; } \\
\text { ECOG-PS } 0 \text { or 1; previous treatment with } \\
\text { Sorafenib (stopped because of progression } \\
\text { or intolerance). }\end{array}$ & $9.2 ; 7.6$ & $2.8 ; 2.1$ & $3.5 ; 2.6$ & $7 ;<1$ & $70.4 ; 54.3$ \\
\hline $\begin{array}{c}\text { CheckMate } 040 \\
{[19,20]}\end{array}$ & Nivolumab & 214 & $\begin{array}{c}\text { Histologically confirmed advanced HCC } \\
\text { (not amenable to curative surgery or local } \\
\text { treatment); Child-Pugh A or B7; ECOG-PS 0 } \\
\text { or 1; disease progression while receiving at } \\
\text { least one previous line of systemic therapy, } \\
\text { including sorafenib or } \\
\text { intolerance to Sorafenib. }\end{array}$ & 15.1 & - & - & 14 & 18.6 \\
\hline $\begin{array}{c}\text { KeyNote-240 } \\
\text { [23] }\end{array}$ & Pembrolizumab; Placebo & $278 ; 135$ & $\begin{array}{l}\text { BCLC C or B refractory or not amenable to } \\
\text { locoregional therapy; Child-Pugh A; } \\
\text { ECOG-PS } 0 \text { or 1; radiographic progression } \\
\text { during or intolerance to sorafenib treatment }\end{array}$ & $13.9 ; 11.6$ & $3.0 ; 2.8$ & $3.8 ; 2.8$ & $18.3 ; 4.4$ & $52.7 ; 46.3$ \\
\hline
\end{tabular}

Objective Response Rate. Mo., months. SAEs, Severe (grade $\geq 3$ ) adverse events. 
Table A2. Model parameters and characteristics of patients.

\begin{tabular}{|c|c|c|}
\hline Variables & Base-Case & References \\
\hline \multicolumn{3}{|l|}{ First-line systemic treatments } \\
\hline \multicolumn{3}{|c|}{ Weibull distribution parameters of PFS (scale; shape) } \\
\hline Sorafenib & $6.48 ; 1.09$ & \\
\hline Lenvatinib & $12.06 ; 1.18$ & \\
\hline Atezolizumab plus Bevacizumab & $9.78 ; 1.2$ & \\
\hline \multicolumn{3}{|c|}{$\begin{array}{c}\text { Mean PFS (SD) (months) obtained with Weibull } \\
\text { distribution }\end{array}$} \\
\hline Sorafenib & $6.5(0.13) \mathrm{mo}$. & \\
\hline Lenvatinib & $10.8(0.44) \mathrm{mo}$. & \\
\hline Atezolizumab plus Bevacizumab & $9.1(0.43)$ mo. & \\
\hline \multicolumn{3}{|l|}{ Characteristics of patients } \\
\hline \multicolumn{3}{|l|}{ Male sex } \\
\hline Sorafenib & $85.5 \%$ & {$[12,14,35-38]$} \\
\hline Lenvatinib & $84.7 \%$ & [12] \\
\hline Atezolizumab plus Bevacizumab & $82.4 \%$ & [14] \\
\hline \multicolumn{3}{|l|}{ Asian ethnicity } \\
\hline Sorafenib & $64.6 \%$ & {$[12,14,35-38]$} \\
\hline Lenvatinib & $69.9 \%$ & [12] \\
\hline Atezolizumab plus Bevacizumab & $39.6 \%$ & [14] \\
\hline \multicolumn{3}{|l|}{$\mathrm{HBV}$} \\
\hline Sorafenib & $39.7 \%$ & {$[12,14,35-38]$} \\
\hline Lenvatinib & $52.5 \%$ & [12] \\
\hline Atezolizumab plus Bevacizumab & $48.8 \%$ & [14] \\
\hline \multicolumn{3}{|l|}{$\mathrm{HCV}$} \\
\hline Sorafenib & $23.4 \%$ & {$[12,14,35-38]$} \\
\hline Lenvatinib & $19.0 \%$ & [12] \\
\hline Atezolizumab plus Bevacizumab & $21.4 \%$ & [14] \\
\hline \multicolumn{3}{|c|}{ Vascular Invasion and/or extrahepatic disease } \\
\hline Sorafenib & $63.3 \%$ & {$[12,14,35-38]$} \\
\hline Lenvatinib & $68.8 \%$ & [12] \\
\hline Atezolizumab plus Bevacizumab & $76.8 \%$ & [14] \\
\hline \multicolumn{3}{|l|}{ ECOG Performance status 1} \\
\hline Sorafenib & $32.0 \%$ & {$[12,14,35-38]$} \\
\hline Lenvatinib & $36.4 \%$ & [12] \\
\hline Atezolizumab plus Bevacizumab & $37.7 \%$ & [14] \\
\hline \multicolumn{3}{|l|}{ BCLC C } \\
\hline Sorafenib & $71.4 \%$ & {$[12,14,35-38]$} \\
\hline Lenvatinib & $78.2 \%$ & [12] \\
\hline Atezolizumab plus Bevacizumab & $82.1 \%$ & [14] \\
\hline \multicolumn{3}{|l|}{ Second-line systemic treatments } \\
\hline \multicolumn{3}{|l|}{ Weibull distribution parameters (scale; shape) } \\
\hline Regorafenib & $15.36 ; 1.23$ & \\
\hline Cabozantinib & $15.24 ; 1.27$ & \\
\hline Ramucirumab & $15.29 ; 1.21$ & \\
\hline Nivolumab & $22.8 ; 1.11$ & \\
\hline Pembrolizumab & $18.96 ; 1.22$ & \\
\hline \multicolumn{3}{|c|}{$\begin{array}{c}\text { Mean OS (SD) (months) obtained with Weibull } \\
\text { distribution }\end{array}$} \\
\hline Regorafenib & $14.8(0.62) \mathrm{mo}$. & \\
\hline Cabozantinib & $14.7(0.54) \mathrm{mo}$. & \\
\hline Ramucirumab & $14.9(0.7) \mathrm{mo}$. & \\
\hline Nivolumab & $22.2(1.34) \mathrm{mo}$. & \\
\hline Pembrolizumab & $18.5(0.87) \mathrm{mo}$. & \\
\hline \multicolumn{3}{|l|}{ SAEs } \\
\hline Regorafenib & $79.7 \%$ & [15] \\
\hline Cabozantinib & $79.4 \%$ & [16] \\
\hline Ramucirumab & $70.4 \%$ & [39] \\
\hline Nivolumab & $18.6 \% *$ & [20] \\
\hline Pembrolizumab & $52.7 \%$ & [23] \\
\hline
\end{tabular}

HCC, Hepatocellular carcinoma. BCLC, Barcelona Clinic Liver Cancer. PS, Performance status. OS, Overall Survival. PFS, Progression-Free Survival. Mo., months. SAEs, Severe (grade $\geq 3$ ) adverse events. 


\section{References}

1. Cabibbo, G.; Enea, M.; Attanasio, M.; Bruix, J.; Craxì, A.; Cammà, C. A meta-analysis of survival rates of untreated patients in randomized clinical trials of hepatocellular carcinoma. Hepatology 2010, 51, 1274-1283. [CrossRef] [PubMed]

2. Villa, E.; Critelli, R.; Lei, B.; Marzocchi, G.; Cammà, C.; Giannelli, G.; Pontisso, P.; Cabibbo, G.; Enea, M.; Colopi, S.; et al. Neoangiogenesis-related genes are hallmarks of fast-growing hepatocellular carcinomas and worst survival. Results from a prospective study. Gut 2016, 65, 861-869. [CrossRef] [PubMed]

3. Villanueva, A. Hepatocellular Carcinoma. N. Engl. J. Med. 2019, 380, 1450-1462. [CrossRef] [PubMed]

4. Llovet, J.M.; Ricci, S.; Mazzaferro, V.; Hilgard, P.; Gane, E.; Blanc, J.F.; de Oliveira, A.C.; Santoro, A.; Raoul, J.L.; Forner, A.; et al. Sorafenib in advanced hepatocellular carcinoma. N. Engl. J. Med. 2008, 359, 378-390. [CrossRef] [PubMed]

5. $\quad$ Cheng, A.L.; Kang, Y.K.; Chen, Z.; Tsao, C.J.; Qin, S.; Kim, J.S.; Feng, J.; Ye, S.; Yang, T.S.; Xu, J.; et al. Efficacy and safety of sorafenib in patients in the Asia-Pacific region with advanced hepatocellular carcinoma: A phase III randomised, double-blind, placebo-controlled trial. Lancet Oncol. 2009, 10, 25-34. [CrossRef]

6. Iavarone, M.; Cabibbo, G.; Piscaglia, F.; Zavaglia, C.; Grieco, A.; Villa, E.; Cammà, C.; Colombo, M.; SOFIA (SOraFenib Italian Assessment) Study Group. Field-practice study of sorafenib therapy for hepatocellular carcinoma: A prospective multicenter study in Italy. Hepatology 2011, 54, 2055-2063. [CrossRef]

7. Cammà, C.; Cabibbo, G.; Petta, S.; Enea, M.; Iavarone, M.; Grieco, A.; Gasbarrini, A.; Villa, E.; Zavaglia, C.; Bruno, R.; et al. Cost-effectiveness of sorafenib treatment in field practice for patients with hepatocellular carcinoma. Hepatology 2013, 57, 1046-1054. [CrossRef]

8. Iavarone, M.; Cabibbo, G.; Biolato, M.; Della Corte, C.; Maida, M.; Barbara, M.; Basso, M.; Vavassori, S.; Craxì, A.; Grieco, A.; et al. Predictors of survival in patients with advanced hepatocellular carcinoma who permanently discontinued sorafenib. Hepatology 2015, 62, 784-791. [CrossRef]

9. Bruix, J.; Cheng, A.L.; Meinhardt, G.; Nakajima, K.; De Sanctis, Y.; Llovet, J. Prognostic factors and predictors of sorafenib benefit in patients with hepatocellular carcinoma: Analysis of two phase III studies. J. Hepatol. 2017, 67, 999-1008. [CrossRef]

10. Reig, M.; Torres, F.; Rodriguez-Lope, C.; Forner, A.; Llarch, N.; Rimola, J.; Darnell, A.; Ríos, J.; Ayuso, C.; Bruix, J. Early dermatologic adverse events predict better outcome in HCC patients treated with sorafenib. J. Hepatol. 2014, 61, 318-324. [CrossRef]

11. Cabibbo, G.; Cucchetti, A.; Cammà, C.; Casadei-Gardini, A.; Celsa, C.; Emanuele Maria, R.G.; Johnson, P.; Ercolani, G. Outcomes of hepatocellular carcinoma patients treated with sorafenib: A meta-analysis of Phase III trials. Future Oncol. 2019, 15, 3411-3422. [CrossRef] [PubMed]

12. Kudo, M.; Finn, R.S.; Qin, S.; Han, K.H.; Ikeda, K.; Piscaglia, F.; Baron, A.; Park, J.W.; Han, G.; Jassem, J.; et al. Lenvatinib versus sorafenib in first-line treatment of patients with unresectable hepatocellular carcinoma: A randomised phase 3 non-inferiority trial. Lancet 2018, 391, 1163-1173. [CrossRef]

13. Cheng, A.L.; Hsu, C.; Chan, S.L.; Choo, S.P.; Kudo, M. Challenges of combination therapy with immune checkpoint inhibitors for hepatocellular carcinoma. J. Hepatol. 2020, 72, 307-319. [CrossRef] [PubMed]

14. Finn, R.S.; Qin, S.; Ikeda, M.; Ikeda, M.; Galle, P.R.; Ducreux, M.; Kim, T.Y.; Kudo, M.; Breder, B.; Merle, P.; et al. Atezolizumab plus Bevacizumab in Unresectable Hepatocellular Carcinoma. N. Engl. J. Med. 2020, 382, 1894-1905. [CrossRef]

15. Bruix, J.; Qin, S.; Merle, P.; Granito, A.; Huang, Y.H.; Bodoky, G.; Pracht, M.; Yokosuka, O.; Rosmorduc, O.; Breder, V.; et al. Regorafenib for patients with hepatocellular carcinoma who progressed on sorafenib treatment (RESORCE): A randomised, double-blind, placebo-controlled, phase 3 trial. Lancet 2017, 389, 56-66. [CrossRef]

16. Abou-Alfa, G.K.; Meyer, T.; Cheng, A.L.; El-Khoueiry, A.B.; Rimassa, L.; Ryoo, B.Y.; Cicin, I.; Merle, P.; Chen, Y.H.; Park, J.W.; et al. Cabozantinib in patients with advanced and progressing hepatocellular carcinoma. N. Engl. J. Med. 2018, 379, 54-63. [CrossRef]

17. Zhu, A.X.; Kang, Y.K.; Yen, C.J.; Finn, R.S.; Galle, P.R.; Llovet, J.M.; Assenat, E.; Brandi, G.; Pracht, M.; Lim, H.Y.; et al. Ramucirumab after sorafenib in patients with advanced hepatocellular carcinoma and increased $\alpha$-fetoprotein concentrations (REACH-2): A randomised, double-blind, placebo-controlled, phase 3 trial. Lancet Oncol. 2019, 20, 282-296. [CrossRef] 
18. US Food \& Drug Administration: FDA Grants Accelerated Approval to Nivolumab for HCC Previously Treated with Sorafenib. Available online: https://www.fda.gov/drugs/resources-information-approved-drugs/fda-grant S-accelerated-approval-nivolumab-hcc-previously-treated-sorafenib (accessed on 25 September 2017).

19. El-Khoueiry, A.B.; Sangro, B.; Yau, T.; Crocenzi, T.S.; Kudo, M.; Hsu, C.; Kim, T.Y.; Choo, S.P.; Trojan, J.; Welling 3rd, T.H.; et al. Nivolumab in patients with advanced hepatocellular carcinoma (CheckMate 040): An open-label, non-comparative, phase 1/2 dose escalation and expansion trial. Lancet 2017, 389, $2492-2502$. [CrossRef]

20. Yau, T.; Hsu, C.; Kim, T.Y.; Choo, S.P.; Kang, Y.K.; Hou, M.M.; Numata, K.; Yeo, W.; Chopra, A.; Ikeda, M.; et al. Nivolumab in advanced hepatocellular carcinoma: Sorafenib-experienced Asian Cohort Analysis. J. Hepatol. 2019, 71, 543-552. [CrossRef]

21. US Food and Drug Administration: FDA Grants Accelerated Approval to Pembrolizumab for Hepatocellular Carcinoma. Available online: https://www.fda.gov/drugs/fda-grants-accelerated-approval-pembrolizumabhepatocellular-carcinoma (accessed on 14 December 2018).

22. Zhu, A.X.; Finn, R.S.; Edeline, J.; Cattan, S.; Ogasawara, S.; Palmer, D.; Verslype, C.; Zagonel, V.; Fartoux, L.; Vogel, A.; et al. Pembrolizumab in patients with advanced hepatocellular carcinoma previously treated with sorafenib (KEYNOTE-224): A non-randomised, open-label phase 2 trial. Lancet Oncol. 2018, 19, 940-952. [CrossRef]

23. Finn, R.S.; Ryoo, B.Y.; Merle, P.; Kudo, M.; Bouattour, M.; Lim, H.Y.; Breder, V.; Edeline, J.; Chao, Y.; Ogasawara, S.; et al. Results of KEYNOTE-240: Phase 3 study of pembrolizumab vs. best supportive care for second-line therapy in advanced hepatocellular carcinoma. J. Clin. Oncol. 2019, 37, 4004. [CrossRef]

24. Cheng, A.L.; Kang, Y.K.; Lin, D.Y.; Park, J.W.; Kudo, M.; Qin, S.; Chung, H.C.; Song, X.; Xu, J.; Poggi, G.; et al. Sunitinib versus sorafenib in advanced hepatocellular cancer: Results of a randomized phase III trial. J. Clin. Oncol. 2013, 31, 4067-4075. [CrossRef] [PubMed]

25. Chow, P.K.H.; Gandhi, M.; Tan, S.B.; Khin, M.W.; Khasbazar, A.; Ong, J.; Choo, S.P.; Cheow, P.C.; Chotipanich, C.; Asia-Pacific Hepatocellular Carcinoma Trials Group; et al. SIRveNIB: Selective internal radiation therapy versus sorafenib in Asia-Pacific patients with hepatocellular carcinoma. J. Clin. Oncol. 2018, 36, 1913-1921. [CrossRef]

26. Kudo, M.; Ueshima, K.; Yokosuka, O.; Ogasawara, S.; Obi, S.; Izumi, N.; Aikata, H.; Nagano, H.; Hatano, E.; SILIUS Study Group; et al. Sorafenib plus low-dose cisplatin and fluorouracil hepatic arterial infusion chemotherapy versus sorafenib alone in patients with advanced hepatocellular carcinoma (SILIUS): A randomised, open label, phase 3 trial. Lancet Gastroenterol. Hepatol. 2018, 3, 424-432. [CrossRef]

27. Vilgrain, V.; Pereira, H.; Assenat, E.; Guiu, B.; Ilonca, A.D.; Pageaux, G.P.; Sibert, A.; Bouattour, M.; Lebtahi, R.; Allaham, W.; et al. Efficacy and safety of selective internal radiotherapy with yttrium-90 resin microspheres compared with sorafenib in locally advanced and inoperable hepatocellular carcinoma (SARAH): An open-label randomised controlled phase 3 trial. Lancet Oncol. 2017, 18, 1624-1636. [CrossRef]

28. Zhu, A.X.; Park, J.O.; Ryoo, B.Y.; Yen, C.J.; Poon, R.; Pastorelli, D.; Blanc, J.F.; Chung, H.C.; Baron, A.D.; Pfiffer, T.E.F.; et al. Ramucirumab versus placebo as second-line treatment in patients with advanced hepatocellular carcinoma following first-line therapy with sorafenib (REACH): A randomised, double-blind, multicentre, phase 3 trial. Lancet Oncol. 2015, 16, 859-870. [CrossRef]

29. Suzman, D.L.; Pelosof, L.; Rosenberg, A.; Avigan, M.I. Hepatotoxicity of immune checkpoint inhibitors: An evolving picture of risk associated with a vital class of immunotherapy agents. Liver Int. 2018, 38, 976-987. [CrossRef]

30. Peeraphatdit, T.B.; Wang, J.; Odenwald, M.A.; Hu, S.; Hart, J.; Charlton, M.R. Hepatotoxicity from immune checkpoint inhibitors: A systematic review and management recommendation. Hepatology 2020, 72, 315-329. [CrossRef]

31. Schnipper, L.E.; Davidson, N.E.; Wollins, D.S.; Tyne, C.; Blayney, D.W.; Blum, D.; Dicker, A.P.; Ganz, P.A.; Hoverman, J.R.; Langdon, R.; et al. American society of clinical oncology statement: A conceptual framework to assess the value of cancer treatment options. J. Clin. Oncol. 2015, 33, 2563-2577. [CrossRef]

32. Stinnett, A.A.; Mullahy, J. Net health benefits: A new framework for the analysis of uncertainty in cost-effectiveness analysis. Med. Decis. Mak. 1998, 18, S68-S80. [CrossRef]

33. Lu, L.C.; Lee, Y.H.; Chang, C.J.; Shun, C.T.; Fang, C.Y.; Shao, Y.Y.; Liu, T.H.; Cheng, A.L.; Hsu, C.H. Increased expression of programmed death-ligand 1 in infiltrating immune cells in hepatocellular carcinoma tissues after sorafenib treatment. Liver Cancer 2019, 8, 110-120. [CrossRef] [PubMed] 
34. Kato, Y.; Tabata, K.; Kimura, T.; Yachie-Kinoshita, A.; Ozawa, Y.; Yamada, K.; Ito, J.; Tachino, S.; Hori, Y.; Matsuki, M.; et al. Lenvatinib plus anti-PD-1 antibody combination treatment activates CD8+ T cells through reduction of tumor-associated macrophage and activation of the interferon pathway. PLOS ONE 2019, 14, e0212513. [CrossRef] [PubMed]

35. Skitzki, J.J.; Repasky, E.A.; Evans, S.S. Hyperthermia as an immunotherapy strategy for cancer. Curr. Opin. Investig. Drugs 2009, 10, 550-558. [PubMed]

36. Weber, J.S.; D'Angelo, S.P.; Minor, D.; Hodi, F.S.; Gutzmer, R.; Neyns, B. Nivolumab versus chemotherapy in patients with advanced melanoma who progressed after anti-CTLA-4 treatment (CheckMate 037): A randomised, controlled, open-label, phase 3 trial. Lancet Oncol. 2015, 16, 375-384. [CrossRef]

37. Kohn, C.G.; Zeichner, S.B.; Chen, Q.; Montero, A.J.; Goldstein, D.A.; Flowers, C.R. Cost-Effectiveness of Immune Checkpoint Inhibition in BRAF Wild-Type Advanced Melanoma. J. Clin. Oncol. 2017, 35, 1194-1202. [CrossRef]

38. Cabibbo, G.; Petta, S.; Barbara, M.; Attardo, S.; Bucci, L.; Farinati, F.; Giannini, E.G.; Negrini, G.; Ciccarese, F.; Rapaccini, G.L.; et al. Hepatic decompensation is the major driver of death in HCV-infected cirrhotic patients with successfully treated early hepatocellular carcinoma. J. Hepatol. 2017, 67, 65-71. [CrossRef] [PubMed]

39. Cabibbo, G.; Celsa, C.; Calvaruso, V.; Petta, S.; Cacciola, I.; Cannavò, M.R.; Madonia, S.; Rossi, M.; Magro, B.; Rini, F.; et al. Direct-acting antivirals after successful treatment of early hepatocellular carcinoma improve survival in HCV-cirrhotic patients. J. Hepatol. 2019, 71, 265-273. [CrossRef]

40. Llovet, J.M.; Montal, R.; Villanueva, A. Randomized trials and endpoints in advanced HCC: Role of PFS as a surrogate of survival. J. Hepatol. 2019, 70, 1262-1277. [CrossRef]

41. Guyot, P.; Ades, A.E.; Ouwens, M.J.; Welton, N.J. Enhanced secondary analysis of survival data: Reconstructing the data from published Kaplan-Meier survival curves. BMC Med. Res. Methodol. 2012, 12, 9. [CrossRef]

(C) 2020 by the authors. Licensee MDPI, Basel, Switzerland. This article is an open access article distributed under the terms and conditions of the Creative Commons Attribution (CC BY) license (http://creativecommons.org/licenses/by/4.0/). 